NOvA's Latest 3-Flavor Neutrino Oscillation Results

Miranda Elkins ${ }^{a}$ lowa State University

(for the NOvA Collaboration)

\section{The NOvA Experiment}

NOvA is a long-baseline neutrino oscillation experiment that sits in front of Fermilab's 700 kW NuMl muon neutrino beam

The experiment is com posed of two functionally identical detectors that:

- Utilize liquid-scintillator

- Are placed $809 \mathrm{~km}$ apart

- Sit 14.6 mrad off-axis from the NuMl beam

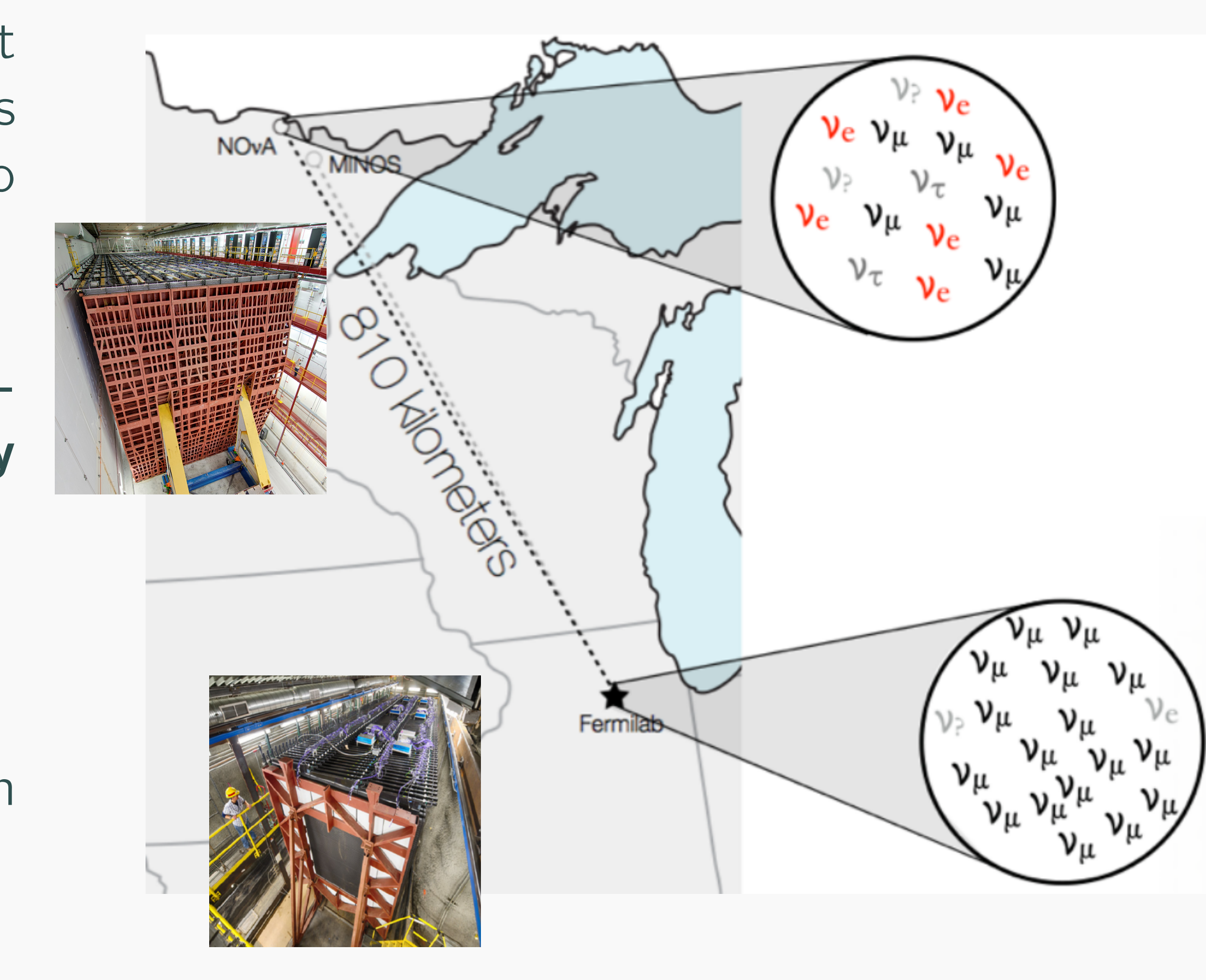

The Physics Goals of NOvA

NOvA uses measurements of $\nu_{\mu}\left(\bar{\nu}_{\mu}\right)$ disappearance $\left(\nu_{\mu} \rightarrow \nu_{\mu}, \bar{\nu}_{\mu} \rightarrow \bar{\nu}_{\mu}\right)$ and $\nu_{e}\left(\bar{\nu}_{e}\right)$ appearance $\left(\nu_{\mu} \rightarrow \nu_{e}, \bar{\nu}_{\mu} \rightarrow \bar{\nu}_{e}\right)$ at the far detector to:

- Resolve the mass hierarchy - Determine the octant of the mixing an(related to appearance) gle $\theta_{23}$ (disappearance and appearance)

- Probe the CP violating phase $\delta_{C}$ (related to appearance) - Constrain the mass squared difference $\Delta m_{32}^{2}$ (related to disappearance)

\section{Observing Neutrinos at the Far Detector}

An example of a $\nu_{\mu}$ interaction in the NOvA FD selected in the analysis.

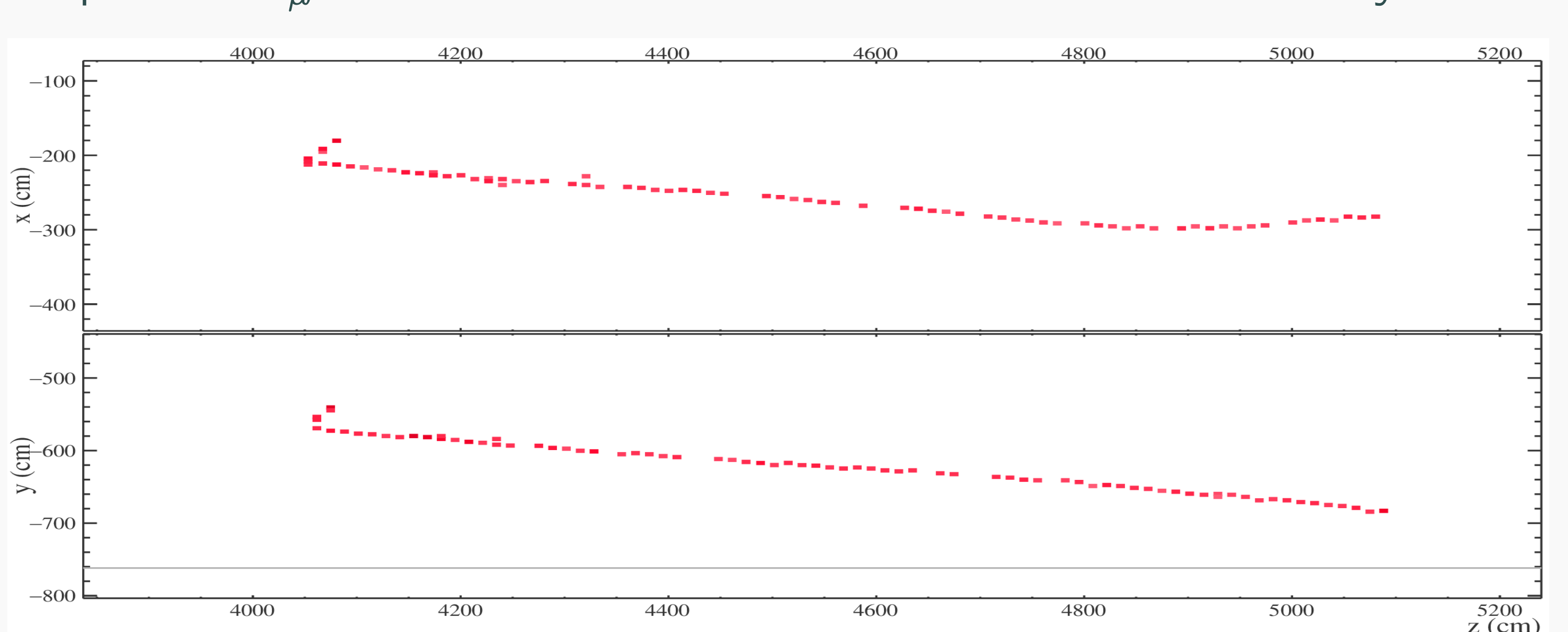

An example of a $\nu_{e}$ interaction in the NOvA FD selected in the analysis.

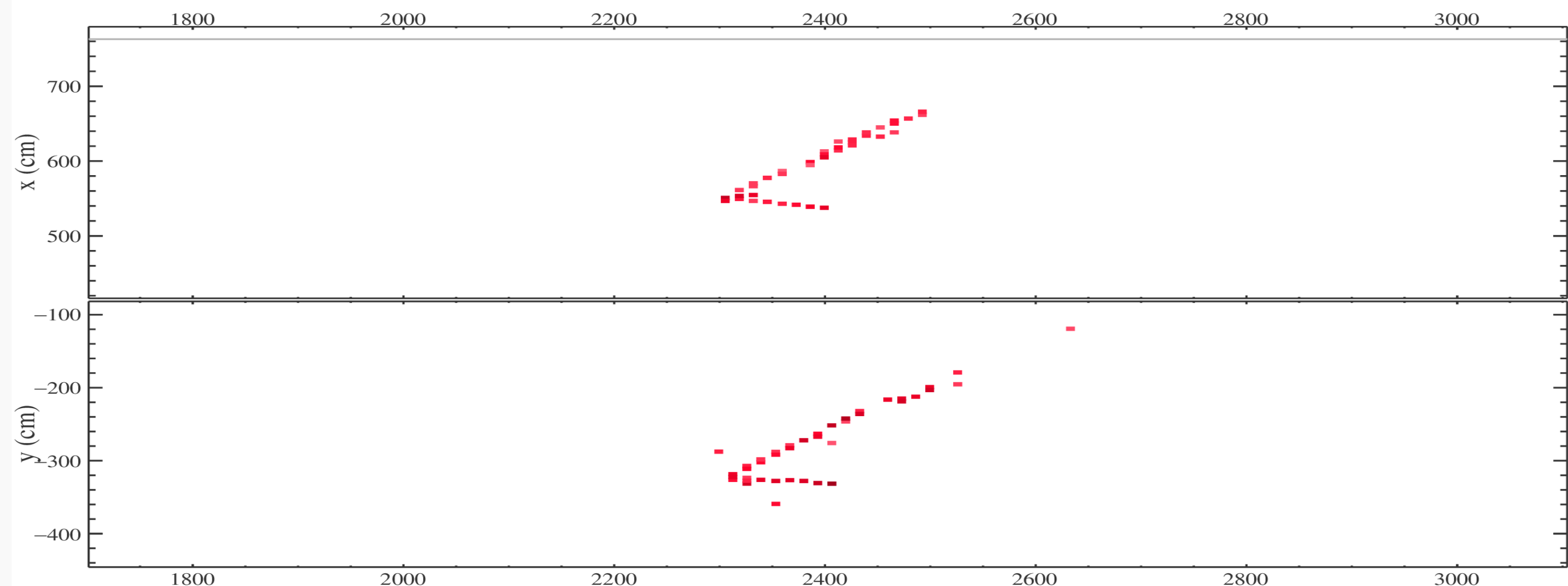

NOvA's $2020 \nu_{\mu}$ and $\bar{\nu}_{\mu}$ Energy Distributions
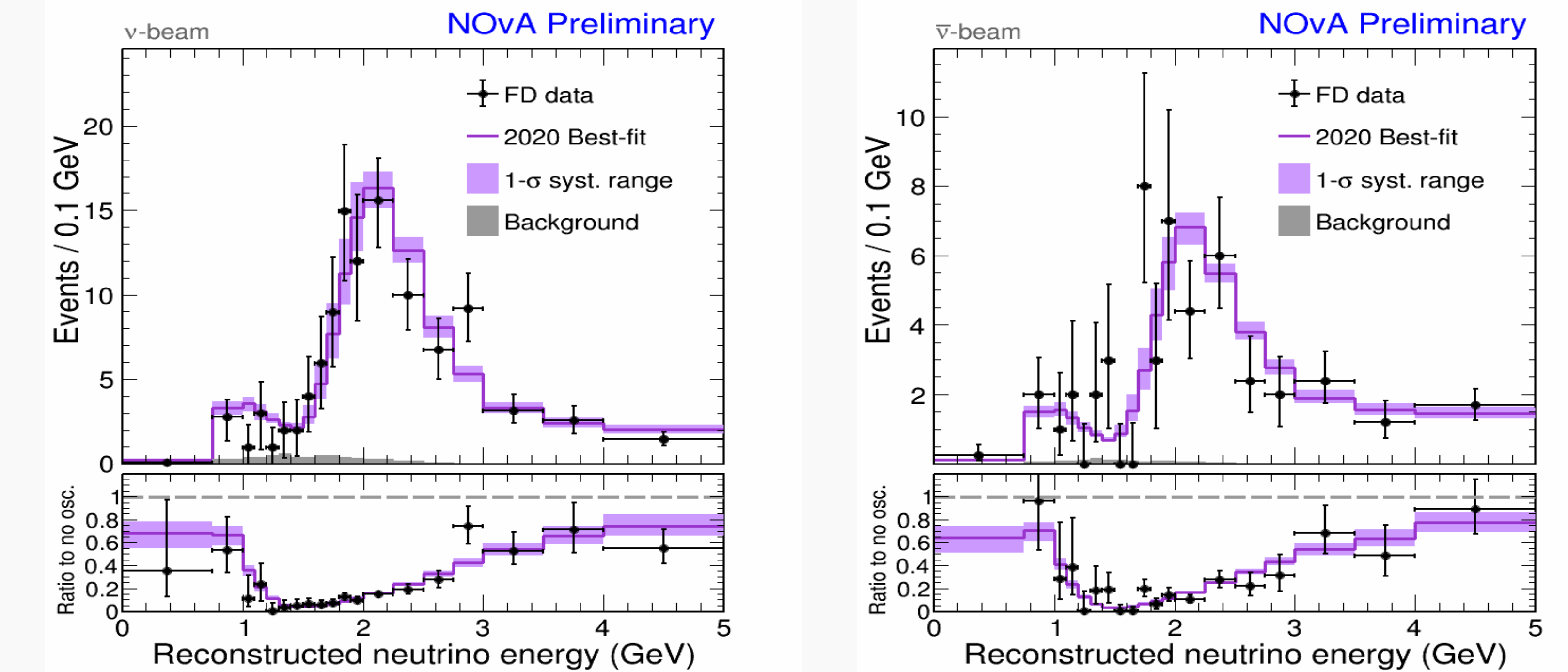

Reconstructed energy of NOVA's $\nu_{\mu}$ and $\bar{\nu}_{\mu}$ FD data along with best-fit predictions. For the 2020 analysis NOvA observed $211 \nu_{\mu}$ and $105 \bar{\nu}_{\mu}$ events with an expected background of 8.2 and 2.1 respectively.

NOvA's $2020 \nu_{e}$ and $\bar{\nu}_{e}$ Energy Distributions
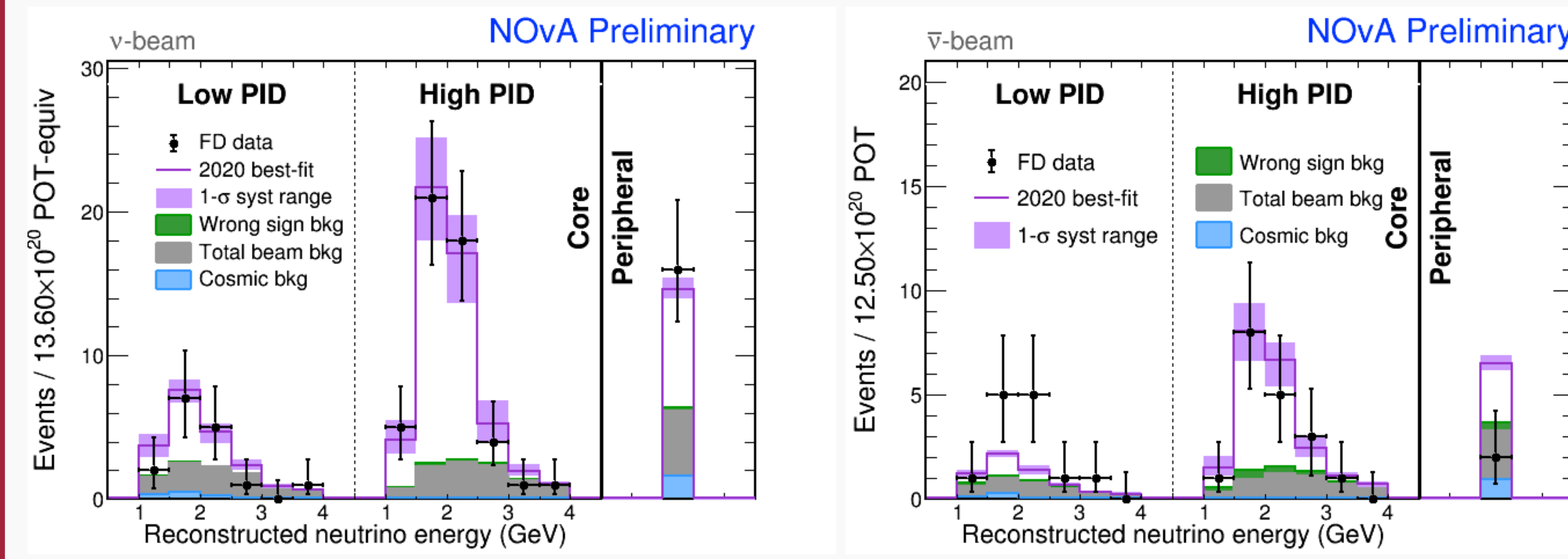

Reconstructed energy of NOvA's $\nu_{e}$ and $\bar{\nu}_{e}$ FD data along with the best-fit predictions. Here the data and predictions are separated into threes bins based on how well the event was identified using a PID and if was contained far from detector edges. expected background of 26.8 and 14 respectively.

\section{Combined $\nu_{e}$ and $\bar{\nu}_{e}$ Results}

The $\mathrm{CP}$ violating phase and the mass hierarchy have opposite effects on the neutrino and antineutrino oscillations so measuring both probabilities helps with resolving the questions we have The distribution shows values of $\delta$ c (ellipses) for combinations of mass hile

The NOvA best fit slightly favors the upper octant and normal hierarchy.

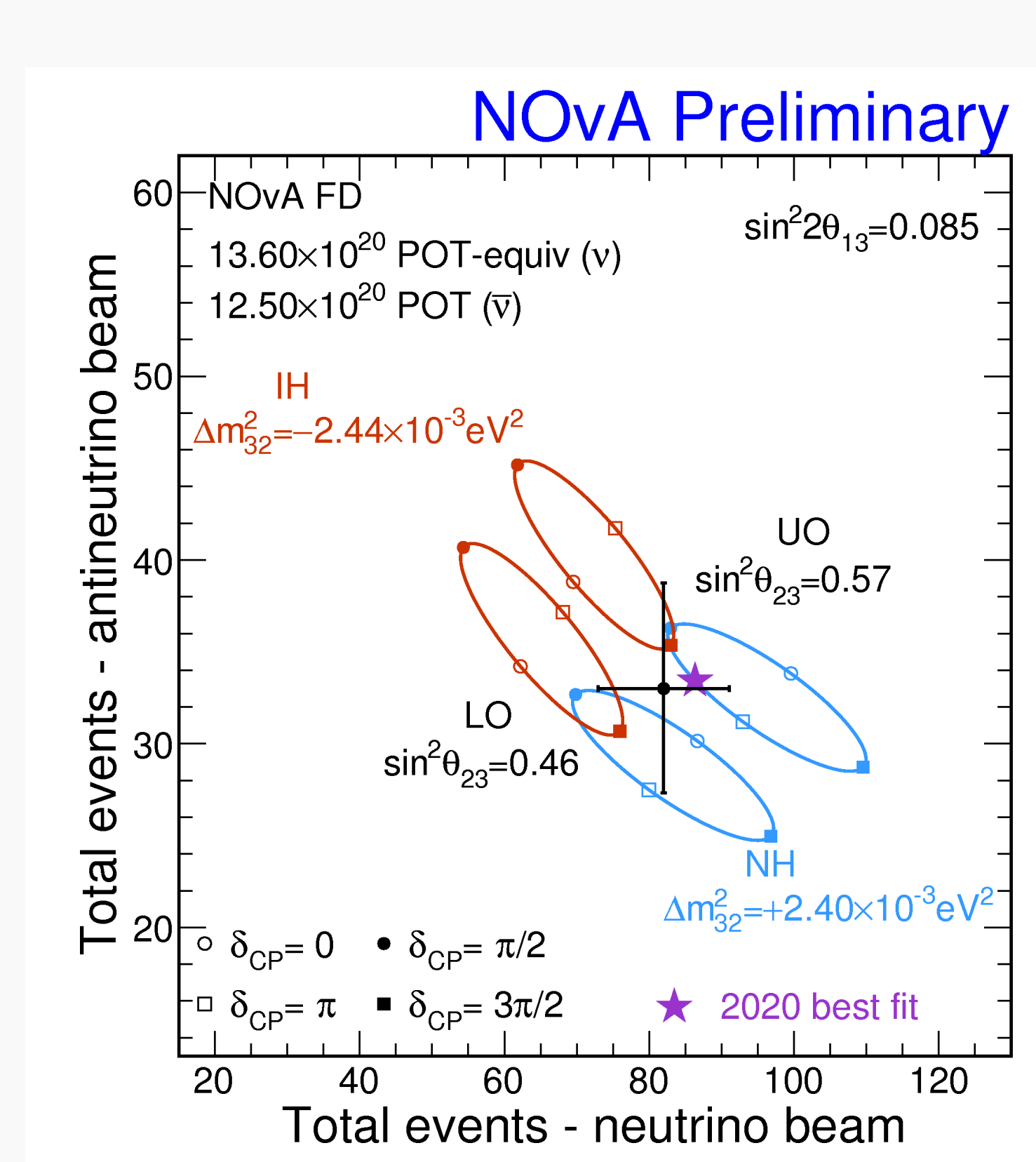

\section{Analysis Best Fits}

The distribution on the left shows the regions of $\delta_{C P}$ and $\sin ^{2} \theta_{23}$ that are within $3 \sigma$ of NOvA's data.

The best fit values fall in the normal hierarchy and are:

- $\delta_{C P}=0.8 \pi$

- $\sin ^{2} \theta_{23}=0.57_{-0.03}^{+0.04}$
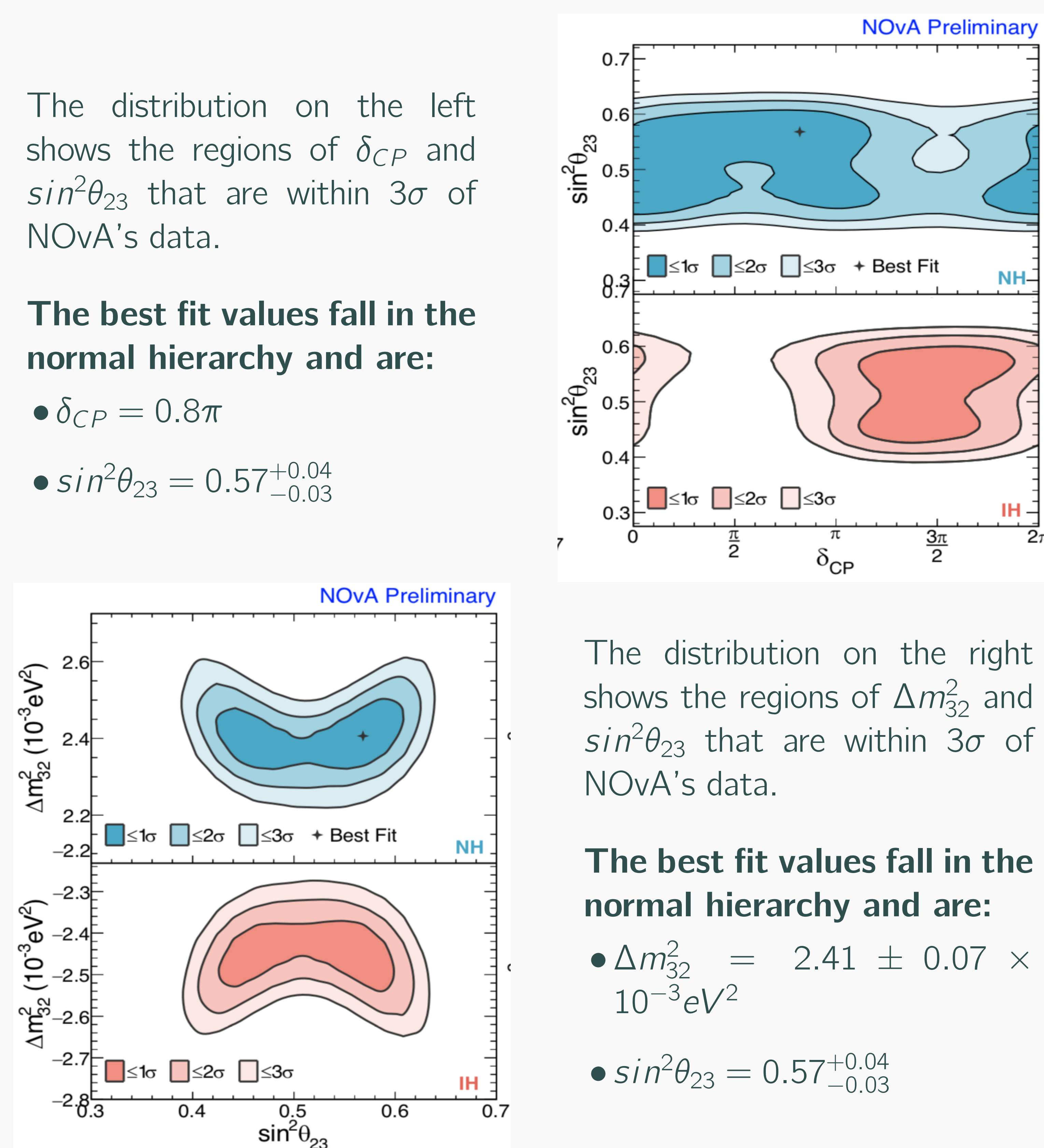

The distribution on the right shows the regions of $\Delta m_{32}^{2}$ and $\sin ^{2} \theta_{23}$ that are within $3 \sigma$ of NOvA's data.

The best fit values fall in the normal hierarchy and are:

- $\Delta m_{32}^{2}=2.41 \pm 0.07 \times$

$10^{-3} \mathrm{eV}^{2}$

- $\sin ^{2} \theta_{23}=0.57_{-0.03}^{+0.04}$

\section{Looking Toward the Future}

NOvA is expected to run through 2025. For the future sensitivities we assume to take $50 \%$ neutrino and $50 \%$ antineutrino data for the remainder of the run resulting in an exposure of $31.5 \times 10^{20} \mathrm{POT}$ for each beam mode.

By the year 2025 we predict:

- $5 \sigma$ sensitivity to the mass hierarchy in the most favorable case $\left(\mathrm{NH}\right.$ and $\left.\delta_{C P}=3 \pi / 2\right)$

- $2 \sigma$ sensitivity to $\mathrm{CP}$ violation determination if $\mathrm{NH}$ - $3 \sigma$ sensitivity to octant determination

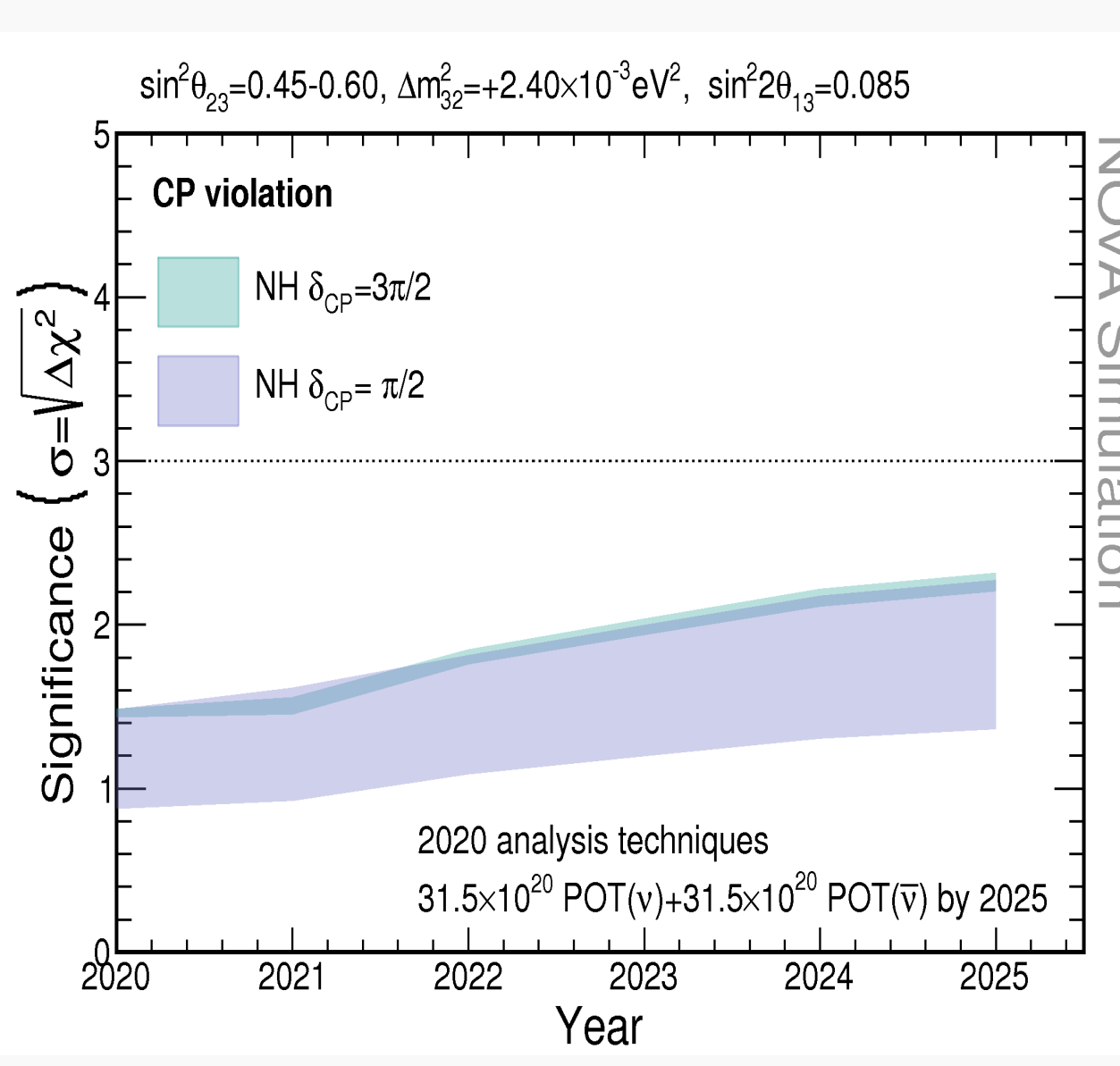

